\title{
A Single Amino Acid Substitution in the Third Transmembrane Region Has Opposite Impacts on the Selectivity of the Parasiticides Fluralaner and Ivermectin for Ligand-Gated Chloride Channels ${ }^{\mathrm{s}}$
}

\author{
Yunosuke Nakata, Toshinori Fuse, Kohei Yamato, Miho Asahi, Kunimitsu Nakahira, \\ Fumiyo Ozoe, and Yoshihisa Ozoe
}

Faculty of Life and Environmental Science, Shimane University, Matsue, Shimane, Japan (Y.N., T.F., K.Y, F.O., Y.O.); and Biological Research Laboratories, Nissan Chemical Industries, Ltd., Saitama, Japan (M.A., K.N.)

Received May 16, 2017; accepted September 9, 2017

\begin{abstract}
Fluralaner (Bravecto) is a recently marketed isoxazoline ectoparasiticide. This compound potently inhibits GABA-gated chloride channels (GABACls) and less potently glutamate-gated chloride channels (GluCls) in insects. The mechanism underlying this selectivity is unknown. Therefore, we sought to identify the amino acid residues causing the low potency of fluralaner toward GluCls. We examined the fluralaner sensitivity of mutant housefly (Musca domestica) GluCls in which amino acid residues in the transmembrane subunit interface were replaced with the positionally equivalent amino acids of Musca GABACls. Of these amino acids, substitution of an amino acid (Leu315) in the third
\end{abstract}

transmembrane region (TM3) with an aromatic amino acid dramatically enhanced the potency of fluralaner in the GluCls. In stark contrast to the enhancement of fluralaner potency, this mutation eliminated the activation of currents and the potentiation but not the antagonism of glutamate responses that are otherwise all elicited by the macrolide parasiticide ivermectin (IVM). Our findings indicate that the amino acid Leu315 in Musca GluCls plays significant roles in determining the selectivity of fluralaner and IVM for these channels. Given the high sequence similarity of TM3, this may hold true more widely for the GluCls and GABACls of other insect species.

\section{Introduction}

Ligand-gated ion channels (LGICs) play vital roles in regulating neuronal excitation and inhibition in animals. These channels are either: 1) cation-selective channels, the activation of which depolarizes the postsynaptic membrane toward firing an action potential; or 2) anion-selective channels, the activation of which hyperpolarizes the membrane or suppresses the depolarization generated by cation channels (Smart and Paoletti, 2012). Nicotinic acetylcholine receptors and GABA receptors, which are members of the Cys-loop family of LGICs, are examples of such cation and anion channels, respectively (Miller and Smart, 2010). Inhibitory glutamate receptors, which are found only in invertebrates, are also Cys-loop LGIC family members. The Cys-loop LGICs are pentamers, the subunits of which are assembled to form a central ion-permeable channel. Each subunit consists of a large N-terminal extracellular domain, four hydrophobic

This work was supported in part by Japan Society for the Promotion of Science [KAKENHI Grant 26292031].

https://doi.org/10.1124/mol.117.109413.

S This article has supplemental material available at molpharm. aspetjournals.org. $\alpha$-helical transmembrane segments (TMs), an intracellular loop between TM3 and TM4, and a short extracellular $\mathrm{C}$ terminus. The orthosteric agonist-binding site is located at the subunit interface of the extracellular domain. These channels are important targets for drugs and insecticides (Ozoe, 2013; Alexander et al., 2015).

Glutamate-gated chloride channels (GluCls) are the main targets for the insecticidal, acaricidal, and nematicidal macrolides avermectins (AVMs), which allosterically activate and modulate various ion channels, including GABAgated chloride channels (GABACls), glycine-gated chloride channels, pH-gated chloride channels, $\alpha 7$ acetylcholinegated cation channels, ATP-gated P2X receptor cation channels, and G protein-gated inwardly rectifying potassium channels (Cully et al., 1994, 1996; Krause et al., 1998; Dawson et al., 2000; Shan et al., 2001; Silberberg et al., 2007; Fuse et al., 2016; Nakatani et al., 2016; Chen et al., 2017). GluCls are activated by nanomolar AVMs, whereas other channels require concentrations in the micromolar range. AVM $B_{1}$ (abamectin) is used to control phytophagous mites and insect pests on agricultural and horticultural crops (Lasota and Dybas, 1991). Ivermectin (IVM) (Fig. 1), 22,23dihydroAVM $\mathrm{B}_{1}$, is widely used to control endoparasites and

ABBREVIATIONS: AVM, avermectin; cRNA, complementary RNA; G36', Gly at position 36'; GABACl, GABA-gated chloride channel; GluCl, glutamate-gated chloride channel; IVM, ivermectin; LGIC, ligand-gated ion channel; PCR, polymerase chain reaction; SOS, standard oocyte solution; TM, transmembrane segment; TSI, transmembrane subunit interface. 
<smiles>Cc1cc(C2=NOC(C)(c3cc(Cl)cc(Cl)c3)C2)ccc1C(=O)NCC(=O)NCC(F)(F)F</smiles>

Fluralaner<smiles>CCCC(=O)NCc1ccc(C2=NOC(C)(c3cc(Br)cc(C(F)(F)F)c3)C2)cc1Cl</smiles>

A341

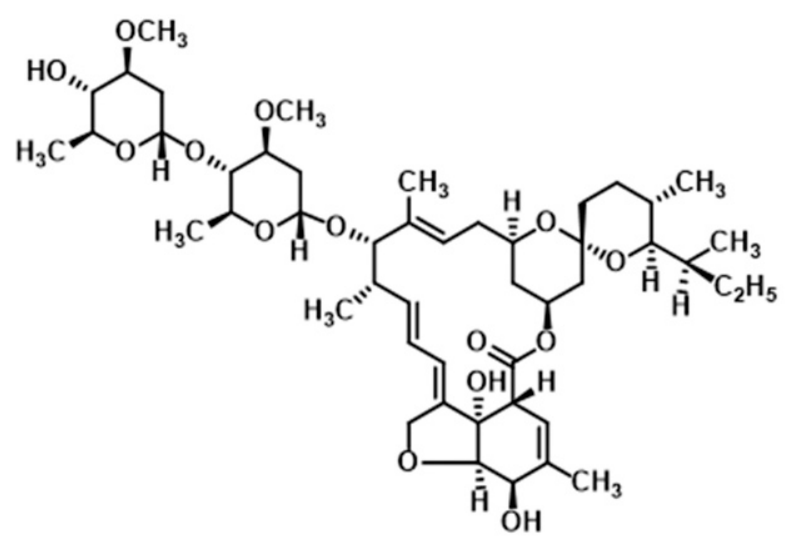

Fig. 1. Chemical structures of fluralaner, its analogs, and IVM $\mathrm{B}_{1 \mathrm{a}}$.

\author{
Ivermectin $\mathrm{B}_{1 \mathrm{a}}$
}

ectoparasites in animals and to treat onchocerciasis and lymphatic filariasis caused by parasitic worms in humans (Ōmura and Crump, 2014; Wolstenholme et al., 2016; Laing et al., 2017). GABACls are targets for chlorinated hydrocarbon and phenylpyrazole insecticides, which stabilize the closed conformation of GABACls by interacting with TM2 amino acid residues on the cytoplasmic side within the channel pore (Ozoe, 2013). Isoxazoline and $m$-benzamidobenzamide insecticides are new-generation antagonists that inhibit insect GABACls (Ozoe et al., 2010, 2013; Nakao et al., 2013; Gassel et al., 2014; Shoop et al., 2014; Asahi et al., 2015; McTier et al., 2016; Nakao and Banba, 2016). These insecticides inhibited GABACls with mutations conferring insensitivity to the conventional antagonists (Nakao et al., 2013; Asahi et al., 2015). The substitution of a conserved TM3 Gly located in the transmembrane subunit interface (TSI) of Drosophila GABACls diminished or eliminated the inhibitory effects of a $m$-benzamidobenzamide (meta-diamide) insecticide on GABA responses, whereas the conventional antagonists remained effective (Nakao et al., 2013). These findings indicate that these insecticides have modes of action distinct from that of the conventional channel-blocking antagonists.

Fluralaner (Bravecto) (Fig. 1) is a recently marketed isoxazoline ectoparasiticide that is used for flea and tick protection in pets (Taenzler et al., 2014; Wengenmayer et al., 2014). This compound inhibits agonist responses in both GABACls and GluCls, with selectivity for the former over the latter (Ozoe et al., 2010), whereas IVM is a selective activator or modulator for GluCls (Fuse et al., 2016). In the present study, we sought to identify the mechanism underlying the difference in fluralaner and IVM sensitivity between GluCls and GABACls. We report that the substitution of a single amino acid in the TSI of GluCls with a positionally equivalent amino acid from GABACls results in a drastic increase in the antagonist potency of fluralaner and a dramatic elimination of the IVM activation of currents and the IVM potentiation of the glutamate responses.

\section{Materials and Methods}

Chemicals. Fluralaner (99\%), A1209 (99\%), and A341 (99\%) were synthesized according to a previously reported method (Mita et al., 2005, 2009, 2010). GABA and IVM (B $\left.\mathrm{B}_{1 \mathrm{a}} \geq 90 \%, \mathrm{~B}_{1 \mathrm{~b}} \leq 5 \%\right)$ were purchased from Sigma-Aldrich (St. Louis, MO). Other general chemicals including sodium hydrogen L-glutamate were purchased from Wako Pure Chemical Industries, Ltd. (Osaka, Japan), unless otherwise noted.

Wild-Type and Mutant GluCl cDNAs. Two LGIC splice variants that show robust agonist responses were used in this study. cDNAs encoding GluCl (variant A) (accession number AB177546) and GABACl (RDL variant ac) (accession numbers AB177547, AB824728, AB824729) subunits from the housefly (Musca domestica; susceptible reference strain from the World Health Organization) were subcloned into the plasmid vectors pcDNA3 and pBluescript $\mathrm{KS}(-)$, respectively (Eguchi et al., 2006; Ozoe et al., 2013). The introduction of mutations into the cDNAs was performed using a QuikChange Site-Directed 
Mutagenesis Kit (Agilent Technologies, Santa Clara, CA) and verified by DNA sequencing.

Two-Electrode Voltage-Clamp Electrophysiology. The lobes of ovaries from female African clawed frogs (Xenopus laevis) anesthetized by immersion in a $0.1 \%(\mathrm{w} / \mathrm{v})$ Tricaine solution were surgically removed. Follicle cells were treated with collagenase $(2 \mathrm{mg} / \mathrm{ml}$; Sigma-Aldrich) in a calcium-free standard oocyte solution $\left(\mathrm{Ca}^{2+}\right.$-free SOS $)(100 \mathrm{mM} \mathrm{NaCl}$, $2 \mathrm{mM} \mathrm{KCl}, 1 \mathrm{mM} \mathrm{MgCl} 2,5 \mathrm{mM}$ HEPES, $\mathrm{pH}$ 7.6) for $1-2$ hours at $20^{\circ} \mathrm{C}$. After the treatment, the oocytes were washed with $\mathrm{Ca}^{2+}$-free SOS supplemented with $2.5 \mathrm{mM}$ sodium pyruvate, gentamycin $(50 \mu \mathrm{g} / \mathrm{ml}$; Thermo Fisher Scientific, Waltham, MA), penicillin (100 U/ml; Thermo Fisher Scientific), and streptomycin (100 $\mu \mathrm{g} / \mathrm{ml}$; Thermo Fisher Scientific) and were incubated for $1-2$ days at $16^{\circ} \mathrm{C}$.

The Musca GluCl and $R d l$ cDNAs containing a T7 promoter site upstream of the coding region were amplified by polymerase chain reaction (PCR). The PCR products were purified using an illustra GFX PCR DNA and Gel Band Preparation Kit (GE Healthcare BioSciences, Pittsburgh, PA). After sequence verification, the amplified cDNA templates ( $100 \mathrm{ng}$ ) were in vitro transcribed into capped poly(A) complementary RNAs (cRNAs) using an mMESSAGE mMACHINE T7 Ultra Kit (Thermo Fisher Scientific). The quality and quantity of the prepared cRNAs were evaluated by agarose gel electrophoresis and absorption spectroscopy, respectively. Purified cRNA (5 ng) was injected into each oocyte using a Nanoliter 2000 injector (World Precision Instruments, Sarasota, FL). The injected oocytes were incubated for $2-4$ days at $16^{\circ} \mathrm{C}$.

The oocytes expressing Musca GluCls or GABACls were placed in a chamber perfused with SOS $(100 \mathrm{mM} \mathrm{NaCl}, 2 \mathrm{mM} \mathrm{KCl}, 1.8 \mathrm{mM}$ $\mathrm{CaCl}_{2}, 1 \mathrm{mM} \mathrm{MgCl}$, $5 \mathrm{mM}$ HEPES, $\mathrm{pH}$ 7.6). Glass microelectrodes were filled with $2 \mathrm{M} \mathrm{KCl}$ to yield a resistance of 0.5-1.6 $\mathrm{M} \Omega$. Electrophysiological recordings were performed using an Oocyte Clamp OC-726C amplifier (Warner Instruments, Hamden, CT) at a holding potential of $-80 \mathrm{mV}$ at $20^{\circ} \mathrm{C}$. The data were digitized using a Laboratory-Trax-4/16 converter (World Precision Instruments) and analyzed using the Data-Trax2 software (World Precision Instruments). IVM, fluralaner, and fluralaner analogs dissolved in dimethyl sulfoxide were diluted with SOS to produce solutions containing the indicated concentrations for each compound and $<0.01 \%$ dimethyl sulfoxide. To analyze the antagonism of the GluCls or GABACls by fluralaner and its analogs, glutamate or GABA dissolved at the $\mathrm{EC}_{50}$ of each channel (Table 1) in SOS was applied to the oocytes for 3 seconds at 30 - to 60 -second intervals with the perfusion of fluralaner until maximum inhibition was achieved. Oocytes were perfused with the solution for 3 minutes to analyze channel activation by IVM. The ability of IVM to potentiate and antagonize glutamate responses was analyzed in a manner similar to the evaluation of fluralaner antagonism but using glutamate at its $\mathrm{EC}_{5}$ and $\mathrm{EC}_{90}$, respectively. The $\mathrm{EC}_{5}$ and $\mathrm{EC}_{90}$ are the concentrations at which the potentiation and antagonism of glutamate responses are observed, respectively (Fuse et al., 2016). All experiments were replicated using at least six oocytes from at least two frogs. The data are presented as the mean \pm S.E.M. The $\mathrm{EC}_{50}$ and $\mathrm{IC}_{50}$ values were obtained from dose-response relationships by the four-parameter logistic regression using OriginPro 8J SR4 (version 8.0951; LightStone, Tokyo, Japan). Unpaired $t$ tests were performed to evaluate statistical significance; $P$ values for L315 mutants are reported with a Bonferroni correction for multiple tests.

Homology Modeling and Docking Simulation. The amino acid sequences of the Musca GluCl-A subunit and the Caenorhabditis elegans GluCl- $\alpha$ subunit were aligned using ClustalW2. A Musca GluCl homology model was constructed using MOE software (version 2014.04; Chemical Computing Group, Montreal, QC, Canada). The X-ray crystal structure of the C. elegans GluCl- $\alpha$ channel (Protein Data Bank code, 3RHW) was used as a template.

\section{Results}

Responses of Wild-Type and Mutant GluCls to Glutamate. The amino acid at position $36^{\prime}$ (index number starting from a conserved TM2 cationic residue numbered $0^{\prime}$ ) in TM3 is particularly important in determining the sensitivity of IVM in LGICs (Lynagh and Lynch, 2010). To examine the effects of intersubunit amino acids on the potencies of fluralaner and IVM against GluCls, we first substituted four GluCl amino acids (Ile253, Met257, Leu315, and Thr316) near Gly at position $36^{\prime}$ (G36') (Gly312 in the Musca GluCl subunit) with the positionally equivalent amino acids of the Musca GABACl (RDL) subunit (Fig. 2), generating four single mutants (I253A, M257L, L315F, and T316V) and one double mutant (M257L/T316V). Because the amino acid at position 253 of the Musca GluCl subunit was identical to the amino acid of the Musca GABACl RDL subunit at the equivalent position, Ile253 was substituted with Ala. All wild-type and mutant GluCls expressed in Xenopus oocytes responded to glutamate to elicit currents (Fig. 3A). The L315F mutant was $\sim 36$-fold less sensitive to glutamate compared with the

TABLE 1

Potencies of glutamate, fluralaner, fluralaner analogs, and IVM in wild-type and mutant forms of Musca GluCls and GABACls expressed in the Xenopus oocytes

\begin{tabular}{llccccc}
\hline Channel & \multicolumn{1}{c}{ Type } & \multicolumn{1}{c}{ Glutamate } & Fluralaner & $\mathrm{A} 1209$ & $\mathrm{~A} 341$ & $\mathrm{IVM}$ \\
\hline & & $\mathrm{EC}_{50}(\mu \mathrm{M})$ & $\mathrm{IC}$ & & \\
GluCl & Wild type & $8.93 \pm 0.21$ & $146 \pm 14$ & $>10,000$ & $>10,000$ & $18.8 \pm 5.0^{a}$ \\
GluCl & I253A & $4.47 \pm 0.32^{* *}$ & $308 \pm 21^{* *}$ & $\mathrm{NT}$ & $\mathrm{NT}$ & $4.01 \pm 1.10^{*}$ \\
GluCl & M257L & $3.32 \pm 0.62^{* *}$ & $39.2 \pm 13.5^{* *}$ & $\mathrm{NT}$ & $\mathrm{NT}$ & $6.91 \pm 2.72$ \\
GluCl & L315F & $323 \pm 57^{*}$ & $1.06 \pm 0.25^{* *}$ & $1.63 \pm 0.49$ & $86.9 \pm 10.2$ & $>3000$ \\
GluCl & L315Y & $81.7 \pm 3.9^{* *}$ & $6.07 \pm 1.27^{* *}$ & $\mathrm{NT}$ & $\mathrm{NT}$ & $>3000$ \\
GluCl & L315W & $\mathrm{NR}$ & $\mathrm{NA}$ & $\mathrm{NA}$ & $\mathrm{NT}$ & $\mathrm{NA}$ \\
GluCl & L315H & $63.3 \pm 9.7^{*}$ & $18.0 \pm 1.6^{* *}$ & $\mathrm{NA}$ & $\mathrm{NT}$ & $>3000$ \\
GluCl & L315M & $12.1 \pm 2.2$ & $77.2 \pm 30.7$ & $\mathrm{NT}$ & $\mathrm{NT}$ & $33.5 \pm 1.5^{*}$ \\
GluCl & L315A & $\mathrm{NR}$ & $\mathrm{NA}$ & $\mathrm{NT}$ & $\mathrm{NT}$ & $\mathrm{NA}$ \\
GluCl & T316V & $17.7 \pm 1.4^{* *}$ & $136 \pm 34$ & $\mathrm{NT}$ & $\mathrm{NT}$ & $20.2 \pm 2.3$ \\
GluCl & M257L/T316V & $9.68 \pm 2.09$ & $65.4 \pm 10.7^{* *}$ & $\mathrm{NT}$ & $\mathrm{NT}$ & $\mathrm{NT}$ \\
GABACl & Wild type & $6.97 \pm 1.21(\mathrm{GABA})$ & $6.05 \pm 1.47$ & $18.8 \pm 6.0$ & $53.2 \pm 9.3$ & $1250 \pm 400^{b}$ \\
\hline
\end{tabular}

NA, not applicable; NR, no response; NT, not tested

${ }^{a} 6.79 \pm 1.48 \mathrm{nM}$ according to Fuse et al. (2016).

${ }^{b}$ Fuse et al. (2016). The data are mean \pm S.E.M. values of six experiments. Unpaired $t$ tests were performed to evaluate statistical significance; $P$ values for L315 mutants are reported with a Bonferroni correction for multiple tests.

$* P<0.05 ; * * P<0.01$ (relative to wild-type GluCl). 
A $\quad 253257$

GIUCI YYLIQIYIPCCMLVIVSWVSFWLDQ

RDL

YYLIQIYIPSGLIVISWVSFWLNR

TM1

B

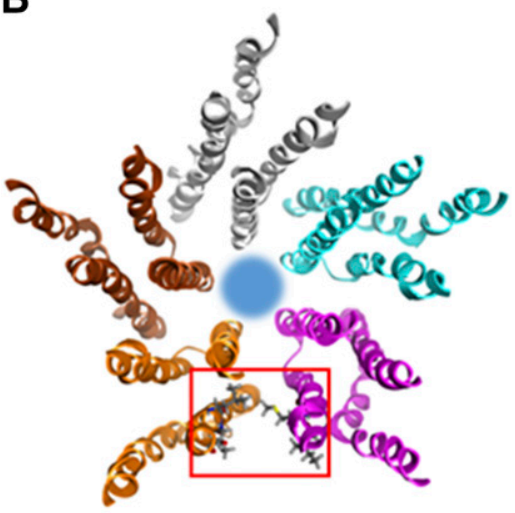

315316

36' II

KAIDVWTGVCLTFVFGALLEFALVNYA

KSIDVYLGTCFVMVFASLLEYATVGYM

TM3

C

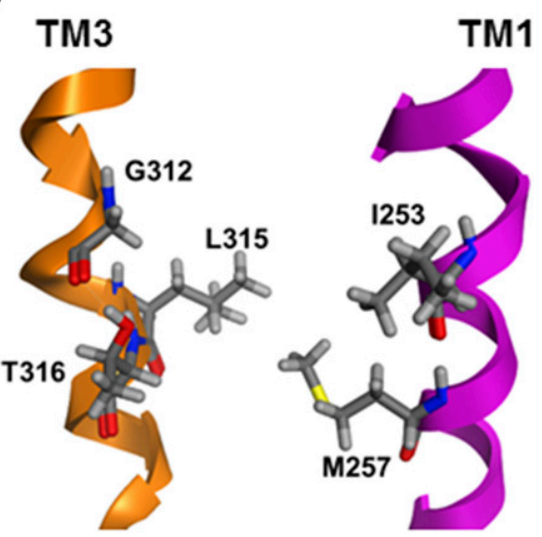

Fig. 2. Location of the amino acid substitution in Musca GluCls. (A) Amino acid alignment of the TM1 and TM3 of Musca GluCl and GABACl (RDL) subunits. Substituted amino acids are highlighted in red. (B) Top view of the channel domain with the side chains of substituted amino acids indicated by highlighting. (C) Close-up of the side chains of substituted amino acids in the TSI. wild-type channel (Table 1). Although this mutation may allosterically affect glutamate binding to the orthosteric site, the dose-response curve indicated that the channel functioned normally to induce currents in response to glutamate.

Fluralaner Inhibition of Glutamate-Induced Currents in Mutant GluCls. Fluralaner inhibited glutamate-induced currents in wild-type Musca GluCls expressed in Xenopus oocytes (Fig. 3, B and D), with an $\mathrm{IC}_{50}$ of $146 \mathrm{nM}$ (Table 1). However, wild-type Musca GluCls were 24-fold less sensitive to fluralaner than were wild-type Musca GABACls, as previously reported (Ozoe et al., 2010). Fluralaner was $\sim 2$-fold less potent toward the I253A mutant than in the wild-type $\mathrm{GluCl}$, whereas it was $\sim 4$-fold and $\sim 2$-fold more potent in the M257L and M257L/T316V mutants, respectively, than in the
A

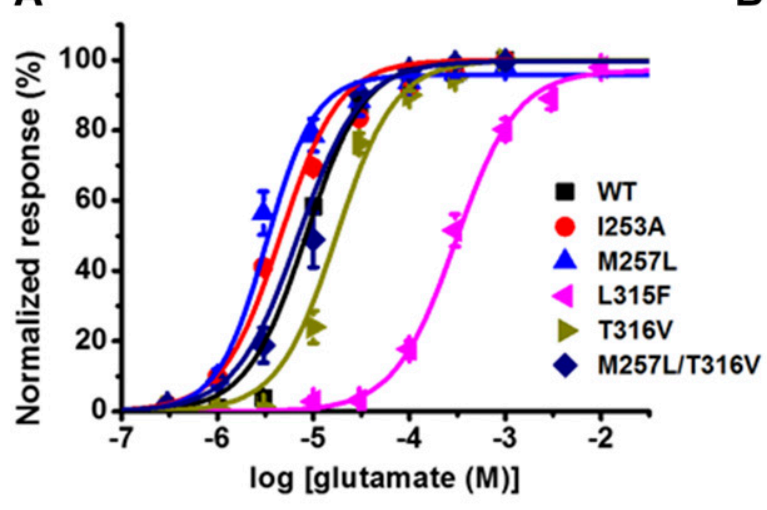

B

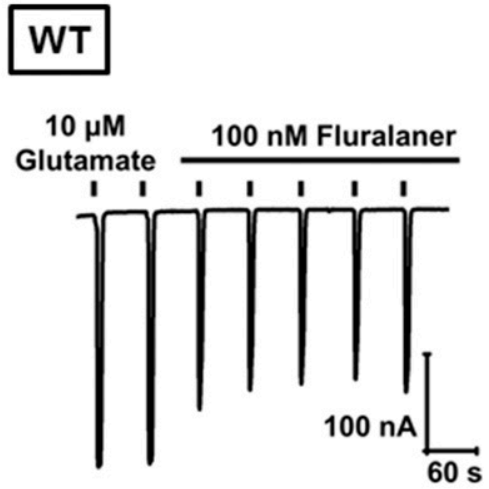

C

\section{L315F}

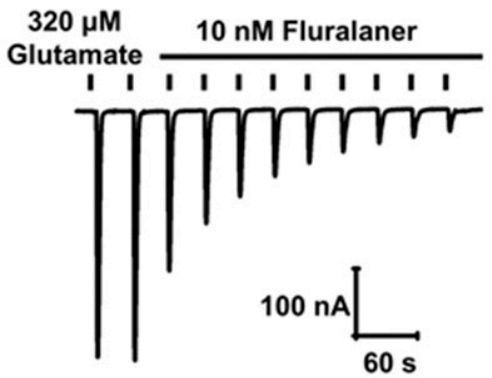

D

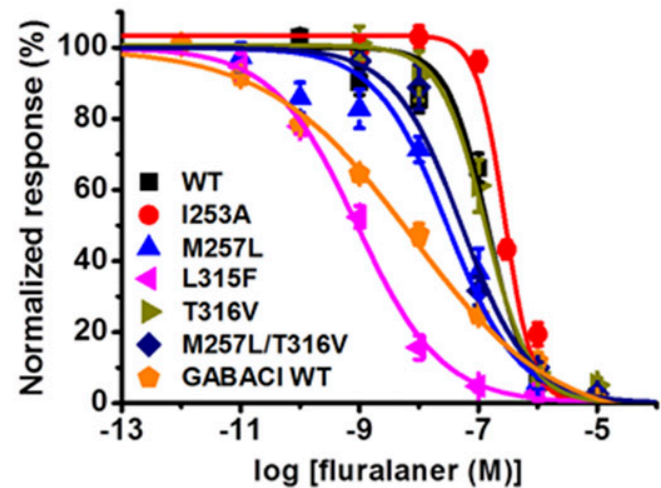

Fig. 3. Inhibition of glutamate-induced currents by fluralaner in wild-type and mutant Musca GluCls. (A) Dose-response curves of glutamate-induced currents. Data points indicate the mean \pm S.E.M. values $(n=6)$ normalized relative to maximal current amplitudes. (B) Current trace of glutamate $\left(\mathrm{EC}_{50}\right)$-induced currents during fluralaner perfusion in the wild-type channel. Note that the slight current recovery in the last glutamate application is within the range encompassed by variation. (C) Current trace of glutamate $\left(\mathrm{EC}_{50}\right)$-induced currents during fluralaner perfusion in the L315F mutant. (D) Dose-response curves of fluralaner inhibition of agonist-induced current in wild-type and mutant GluCls compared with that in the wild-type GABACl. Normalized relative to responses induced by the $\mathrm{EC}_{50}$ values of agonists. Data points indicate mean \pm S.E.M. values $(n=6)$. 
wild-type GluCl (Fig. 3D; Table 1). The potency of fluralaner in the T316V mutant did not differ significantly from that in the wild type. Notably, the L315F mutant was strongly inhibited by fluralaner, with an $\mathrm{IC}_{50}$ of $1.06 \mathrm{nM}$, indicating that this mutant is $\sim 138$-fold more sensitive to fluralaner inhibition than the wild type (Fig. 3, C and D; Table 1). The $\mathrm{IC}_{50}$ of fluralaner in the L315F mutant was even $\sim 6$-fold smaller than its $\mathrm{IC}_{50}$ in the inhibition of GABA-induced currents in Musca GABACls (Fig. 3D; Table 1).

IVM Actions on Mutant GluCls. IVM alone activated slow, irreversible currents in wild-type Musca GluCls expressed in Xenopus oocytes (Fig. 4A), with an $\mathrm{EC}_{50}$ of $18.8 \mathrm{nM}$ (Table 1). The wild-type Musca GluCls were $\sim 66$-fold to 184 -fold more sensitive to IVM than were wild-type Musca GABACls (Table 1). The potency of IVM was $~ 5$-fold higher in the I253A mutant than in the wild type, whereas the potencies of the $\mathrm{M} 257 \mathrm{~L}$ and $\mathrm{T} 316 \mathrm{~V}$ mutants were not significantly different from that of the wild-type GluCl (Fig. $4 \mathrm{C}$; Table 1). The difference in the maximal current amplitude between the wild type and mutants could be ascribed to differences in the expression levels of channels in oocytes (Fig. 4C). Surprisingly, the L315F mutant, which showed an enhanced sensitivity to fluralaner, lacked sensitivity to IVM in terms of activation (Fig. 4, B and C). Next, we examined IVM potentiation and antagonism of glutamate-induced currents in the L315F mutant because IVM exerts a triple action on GluCls and GABACls depending on the conditions (Fuse et al., 2016). In L315F GluCls, IVM did not potentiate the currents induced by a low concentration of glutamate $\left(\mathrm{EC}_{5}, 50 \mu \mathrm{M}\right)$ (Fig. 5, A and B) but inhibited those induced by a high concentration of glutamate $\left(\mathrm{EC}_{90}, 3 \mathrm{mM}\right)$, with an $\mathrm{IC}_{50}$ ( \pm S.E.M.) of $5.48 \pm 1.20 \mathrm{nM}(n=6)$, which is not significantly different from the $\mathrm{IC}_{50}(4.92 \pm 2.23 \mathrm{nM})$ for the wild type (Fig. 5, C and D).

Effects of Fluralaner Analogs on L315F Mutant GluCls. As fluralaner showed marked antagonism of the $\mathrm{L} 315 \mathrm{~F} \mathrm{GluCl}$, we examined whether a similar potency enhancement could be observed for fluralaner analogs (Figs. 6 and 7). The isoxazolines A1209 and A341 (Fig. 1) are fluralaner analogs that show $>500$-fold and $>100$-fold higher antagonism of Musca GABACls, respectively, compared with Musca GluCls (Fig. 6, A, B, and D; Fig. 7, A, B, and D; Table 1). The L315F mutation resulted in $>6000$-fold and $>100$-fold enhancement of the potency of A1209 and A341 in GluCls (Fig. 6, C and D; Fig. 7, C and D; Table 1), which were, respectively, much greater than and comparable to the enhancement observed for fluralaner. The $\mathrm{L} 315 \mathrm{~F} \mathrm{GluCl}$ was outstandingly sensitive to A1209 (Table 1). Although the isoxazoline A341 exhibited little antagonism in wild-type GluCls, the antagonist potency of this compound in L315F GluCls was comparable to that in wild-type GABACls (Fig. 7).

Effects of Aromatic Amino Acid Substitution at Position 315 on the Potency of Fluralaner. As the L315F substitution enhanced the potency of fluralaner against Musca GluCls, we examined the effects of other amino acid substitutions on the potency of fluralaner (Fig. 8). We injected cRNAs encoding five mutants (L315Y, L315W, L315H, L315A, and L315M) into oocytes. The aromatic amino acids included Tyr, Trp, and His, which has the aromatic heterocycle imidazole in the side chain. Ala and Met were
A
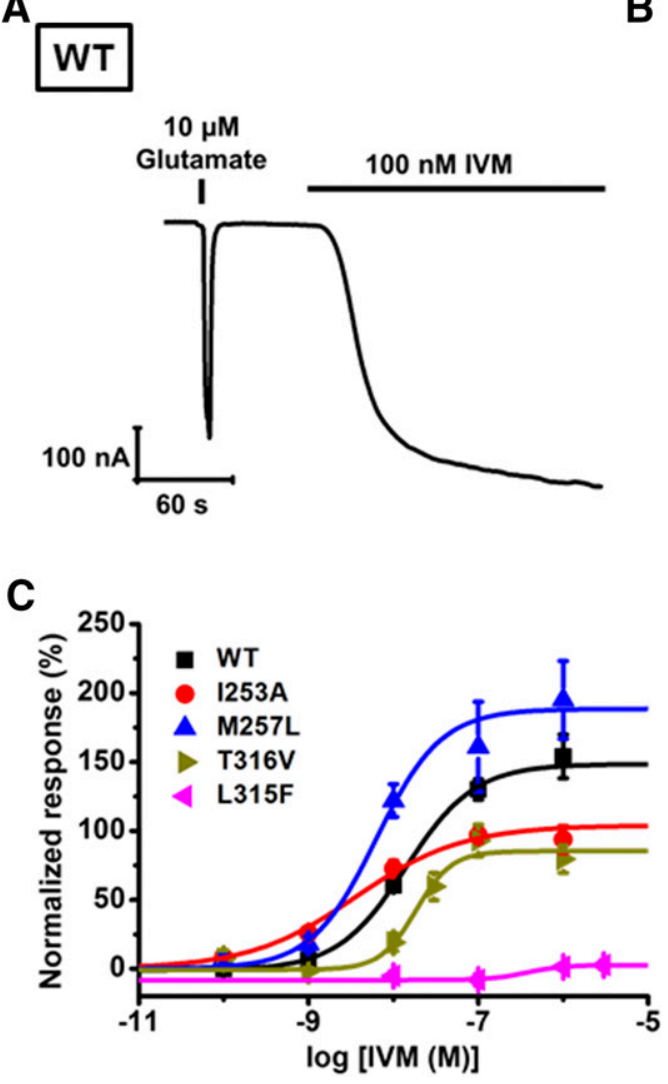

B



Fig. 4. Responses of wild-type and mutant Musca GluCls to IVM. (A) Current trace showing the IVM activation of wild-type GluCls. (B) Current trace when IVM was applied to the L315F mutant. (C) Doseresponse curves of IVM-induced currents in GluCls normalized relative to responses induced by the $\mathrm{EC}_{50}$ values of glutamate. Data points indicate mean \pm S.E.M. values $(n=6)$. 
A

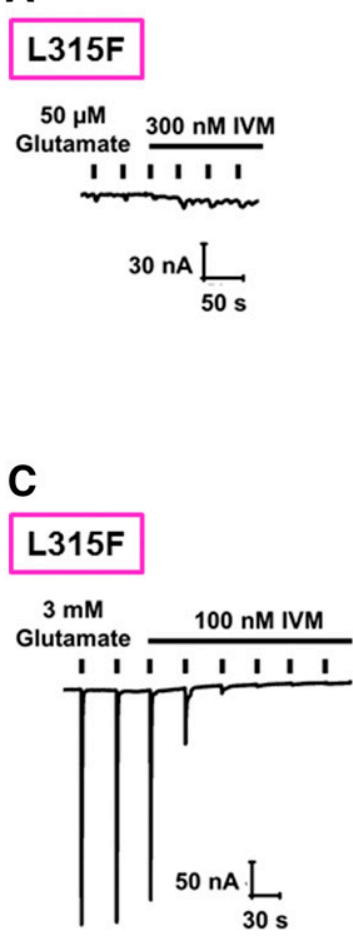

B

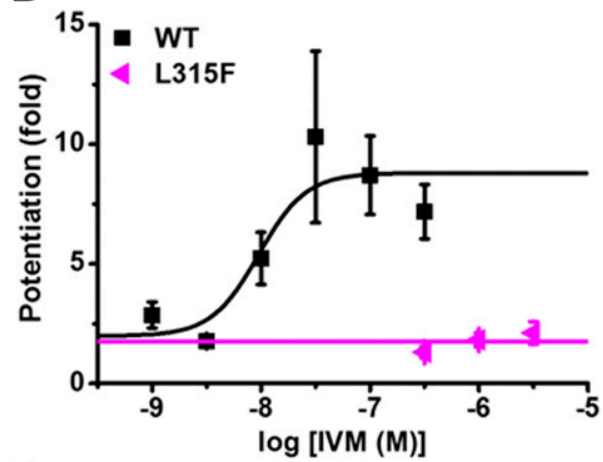

D

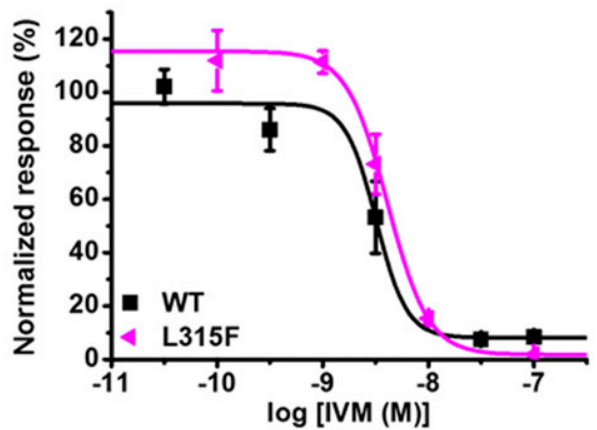

Fig. 5. Responses of wild-type and mutant Musca GluCls to IVM. (A) Current trace showing the absence of the IVM potentiation of glutamate $\left(\mathrm{EC}_{5}\right)$ responses in L315F GluCls. (B) Dose-response curves for evaluating the IVM potentiation of glutamate responses in wild-type and L315F GluCls compared with responses induced by glutamate $\left(\mathrm{EC}_{5}\right)$ alone. Data points indicate mean \pm S.E.M. values $(n=6)$. (C) Current trace showing the IVM inhibition of glutamate $\left(\mathrm{EC}_{90}\right)$-induced currents in the L315F mutant. (D) Dose-response curves of the IVM antagonism of wild-type and L315F GluCls normalized relative to responses induced by the $\mathrm{EC}_{90}$ values of glutamate. Data points indicate mean \pm S.E.M. values $(n=6)$, chosen as hydrophobic aliphatic amino acids. Although the oocytes injected with the cRNAs for L315W and L315A failed to respond to glutamate, the other oocytes did respond to glutamate (Fig. 8A). Fluralaner inhibition of glutamate-induced currents was $\sim 24$-fold and $\sim 8$-fold more potent in the L315Y and L315H mutants, respectively, than in the wild type (Fig. 8, B, C, and E; Table 1). In contrast, the potency of fluralaner did not differ significantly between
A

\section{GABACI WT}

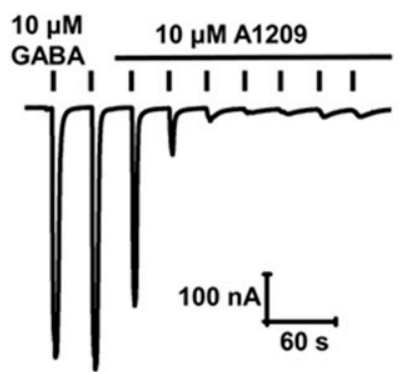

C

\section{GluCI L315F}

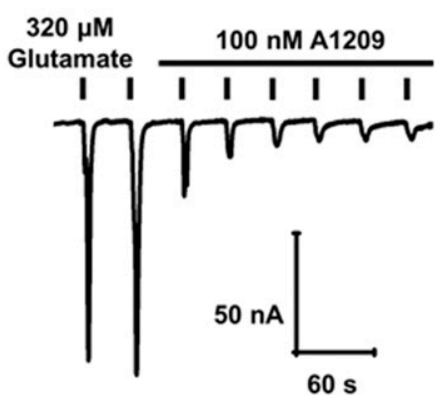

B

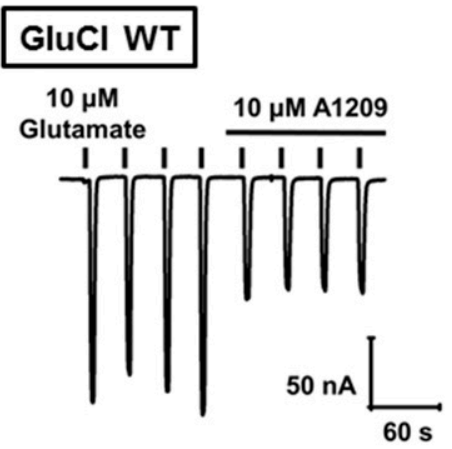

D

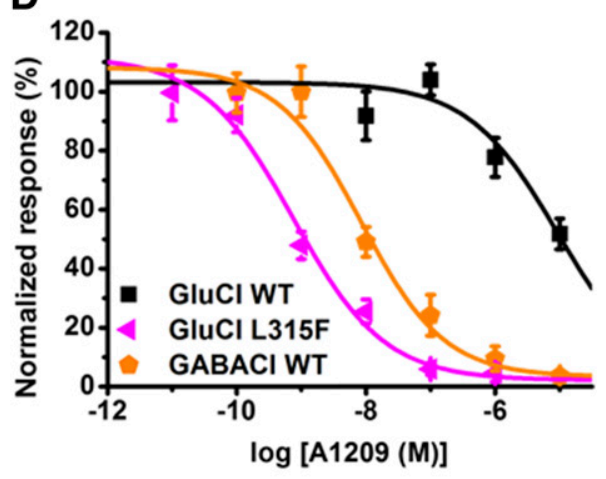

Fig. 6. Inhibition of glutamate-induced currents by a fluralaner analog, A1209, in wild-type and mutant Musca GluCls. (A) Current trace showing the inhibition of GABA $\left(\mathrm{EC}_{50}\right)$-induced currents in wild-type GABACls. (B) Current trace showing the inhibition of glutamate $\left(\mathrm{EC}_{50}\right)$-induced currents in wild-type GluCls. (C) Current trace showing the inhibition of glutamate $\left(\mathrm{EC}_{50}\right)$-induced currents in L315F GluCls. (D) Doseresponse curves of the A1209 inhibition of glutamateand GABA-induced currents normalized relative to responses induced by the $\mathrm{EC}_{50}$ values of agonists. Data points indicate mean \pm S.E.M. values $(n=6)$. 
A

\section{GABACI WT}

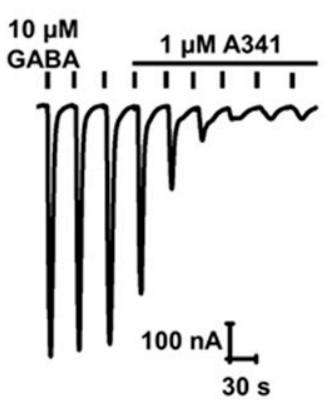

C



B

\section{GluCl WT}

\begin{tabular}{ll}
$10 \mu \mathrm{M}$ & $10 \mu \mathrm{M} \mathrm{A341}$ \\
\cline { 3 - 3 } &
\end{tabular}

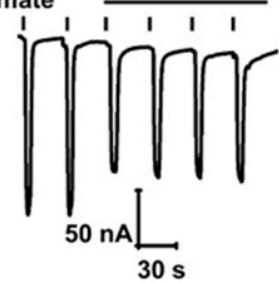

D

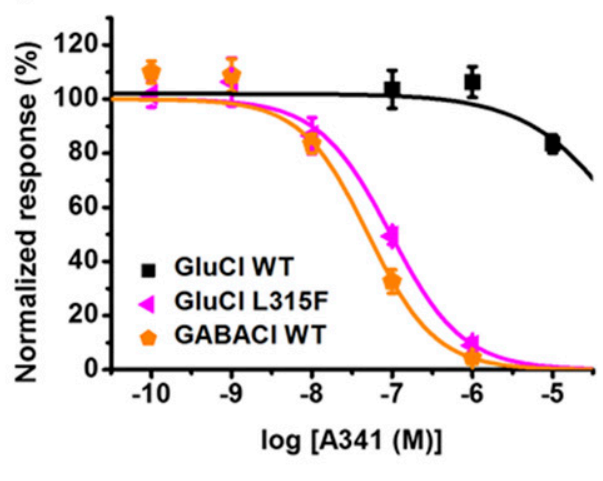

Fig. 7. Inhibition of glutamate-induced currents by a fluralaner analog, A341, in wild-type and mutant Musca GluCls. (A) Current trace showing the inhibition of GABA $\left(\mathrm{EC}_{50}\right)$-induced currents in wild-type GABACls. (B) Current trace showing the inhibition of glutamate $\left(\mathrm{EC}_{50}\right)$-induced currents in wild-type GluCls. (C) Current trace showing the inhibition of glutamate $\left(\mathrm{EC}_{50}\right.$ )-induced currents in L315F GluCls. (D) Dose-response curves of the A341 inhibition of glutamate- and GABA-induced currents normalized relative to responses induced by the $\mathrm{EC}_{50}$ values of agonists. Data points represent mean \pm S.E.M. values $(n=6)$. the L315M mutant and the wild-type channel (Fig. 8, D, E; Table 1). These findings indicate that aromatic amino acids at position 315 are effective in enhancing the antagonist potency of fluralaner in Musca GluCls but that an aliphatic amino acid is not.

Effects of Aromatic Amino Acid Substitution at Position 315 on the Potency of IVM. We examined the effects of the L315Y, L315H, and L315M substitutions on IVM-induced currents in Musca GluCls (Fig. 9). The former two substitutions abolished the IVM-induced activation of Musca GluCls (Fig. 9, A, B, and D). In contrast, IVM activated currents in the L315M mutant, although the potency was $\sim 2$-fold lower than in the wild-type channel (Fig. 7, C and D; Table 1). These findings indicate that aromatic amino acids, but not an aliphatic amino acid, at position 315 eliminate the IVM-induced activation of GluCls.

\section{Discussion}

The TSI of pentameric LGICs plays critical roles in the actions of insecticides and other drugs (Nakao et al., 2013; Forman and Miller, 2016). The TSI in mammalian GABACls has been extensively studied as a binding site for general anesthetics such as propofol, etomidate, and barbiturates (Forman and Miller, 2016). Interestingly, both convulsive and anesthetic barbiturates modulate GABACls by binding to this region (Jayakar et al., 2015). IVM activates currents and potentiates and antagonizes the agonist-induced currents of LGICs including GABACls and GluCls, with the latter being more sensitive than the former, by possibly binding in the TSI (Hibbs and Gouaux, 2011; Estrada-Mondragon and Lynch, 2015; Fuse et al., 2016). A single TM3 amino acid in the TSI, G36' (Fig. 2), which is conserved in IVM-sensitive LGICs, has been shown to be critical for these actions of IVM (Lynagh and Lynch, 2010; Fuse et al., 2016). The substitution of G36' with bulkier amino acids results in a reduction or the loss of sensitivity to IVM. The importance of G36' in insecticide actions was indicated by a report that a G36'D mutation was identified in the abamectin-resistant strain of two-spotted spider mites (Tetranychus urticae) (Kwon et al., 2010). A G36'E substitution disrupted T. urticae GluCl activation by abamectin and milbemycin (Mermans et al., 2017). Furthermore, G36' mutations were reported to diminish or eliminate the ability of a meta-diamide insecticide to inhibit GABA-induced currents in the Drosophila GABACls (Nakao et al., 2013). Interestingly, the analogous mutation of a positionally equivalent Gly was identified in the nicotinic acetylcholine receptor $\alpha 7$ subunit of spinosadresistant western flower thrips (Frankliniella occidentalis) and the nicotinic acetylcholine receptor $\alpha 6$ subunit of spinosad-resistant tomato leafminers (Tuta absoluta) (Puinean et al., 2013; Silva et al., 2016). Together, these reports indicate that a conserved Gly in TM3 plays a key role and that the TSI seems to form the sites of action for a variety of ligands.

The ectoparasiticide fluralaner was shown to exhibit selective antagonism of GABACls over GluCls (Ozoe et al., 2010). To examine whether any amino acid substitution enhances the low potency of fluralaner in GluCls, we focused 
A

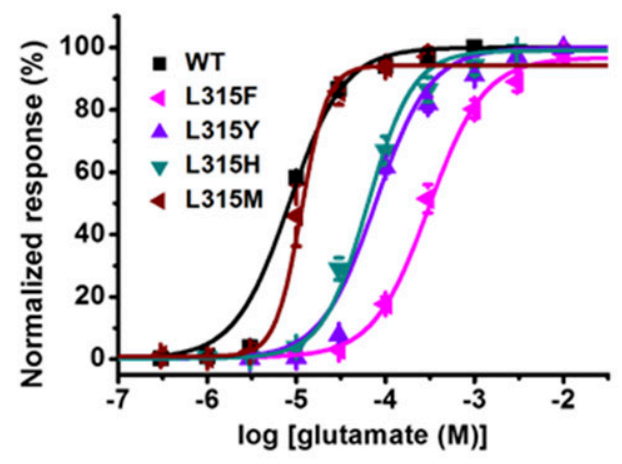

C

\section{L315H}

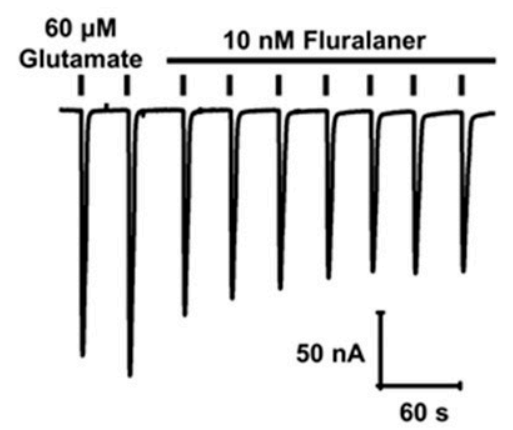

E

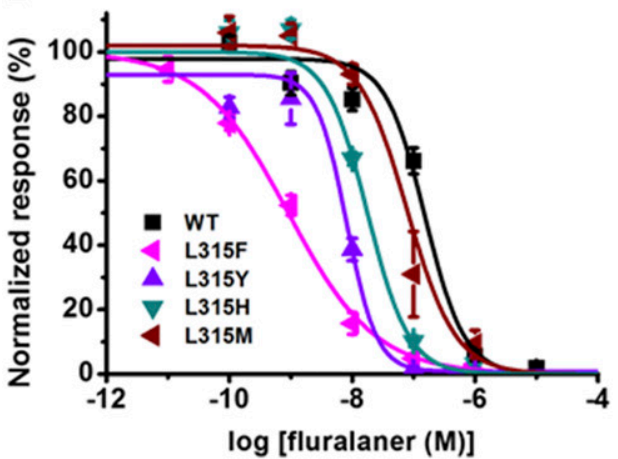

B

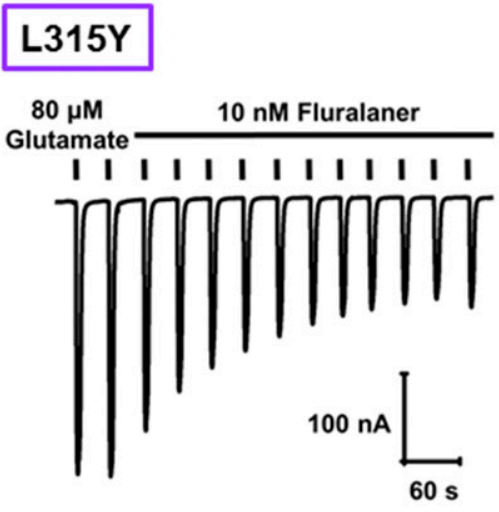

D

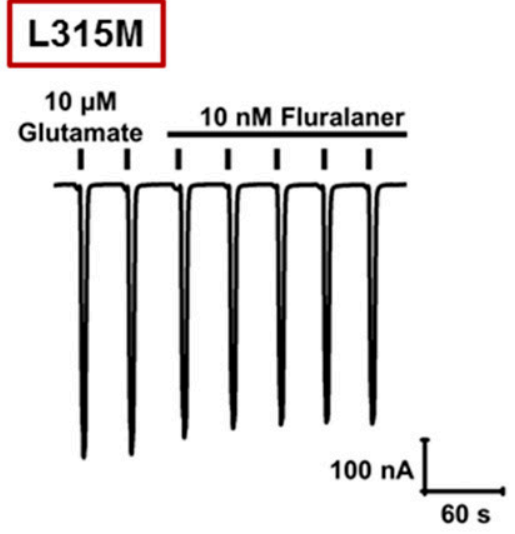

Fig. 8. Inhibition of glutamate-induced currents by fluralaner in Musca L315 mutant GluCls. (A) Dose-response curves of glutamate-induced currents in wild-type and mutant GluCls normalized relative to maximal current amplitudes. Data points indicate mean \pm S.E.M. values $(n=6)$. (B) Current trace of glutamate $\left(\mathrm{EC}_{50}\right)$-induced currents during fluralaner perfusion in the L315Y mutant. (C) Current trace of glutamate $\left(\mathrm{EC}_{50}\right)$-induced currents during fluralaner perfusion in the L315H mutant. (D) Current trace of glutamate $\left(\mathrm{EC}_{50}\right)$-induced current during fluralaner perfusion in the L315M mutant. (E) Dose-response curves of fluralaner inhibition of glutamate-induced currents in wild-type and mutant GluCls normalized relative to responses induced by the $\mathrm{EC}_{50}$ values of glutamate. Data points indicate mean \pm S.E.M. values $(n=6)$. on replacing amino acids around G36' in the TSI of Musca GluCls with the positionally equivalent amino acids of Musca GABACls, which were sensitive to fluralaner (Table 1). We have shown that the substitution of an amino acid, Leu315, located one $\alpha$-helical turn below G36', with aromatic amino acids (but not with an aliphatic amino acid) dramatically enhances the potency of fluralaner (Figs. 3 and 8). These findings may explain the high potency of fluralaner in GABACls, which possess Phe at the equivalent position. Our docking simulation of the $S$ enantiomer of fluralaner, which is the active component (Ozoe et al., 2010), to a Musca $\mathrm{GluCl}$ homology model revealed that the aromatic ring of fluralaner lies near Leu315 (Fig. 10). It remains to be clarified whether the enhanced potency depends on a $\pi-\pi$ stacking interaction (Zhao et al., 2015) between the aromatic ring of substituted amino acids at position 315 and the phenyl group of fluralaner.

In contrast to the enhancement of the potency of fluralaner, we found that the same aromatic substitution abolished the direct IVM activation of GluCls and the IVM potentiation of glutamate-induced currents, while the antagonism remained unchanged (Figs. 4, 5, and 9). The L315M mutant, which has a nonaromatic amino acid at position 315 , retained the ability to be activated by IVM. This finding is consistent with the fact that the homomeric AVM-sensitive $C$. elegans GluCl- $\alpha$ channel has Met at an equivalent position, whereas the AVM-insensitive $\beta$ channel has Gln at this position (Cully et al., 1994) (Supplemental Fig. 1). It will be interesting to investigate whether this Gln is responsible for the insensitivity to AVMs. The amino 
A

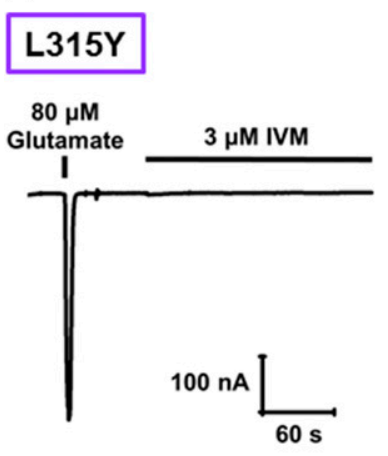

C

\section{L315M}

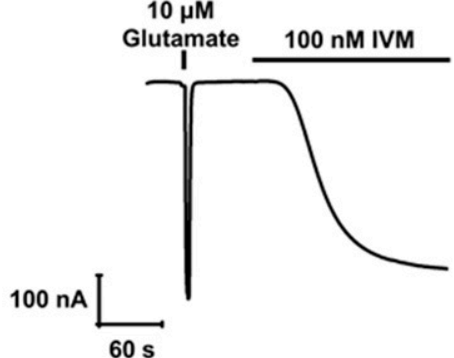

B

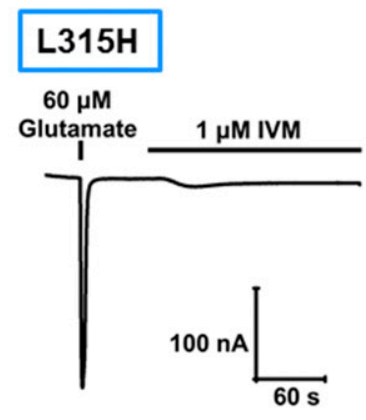

D

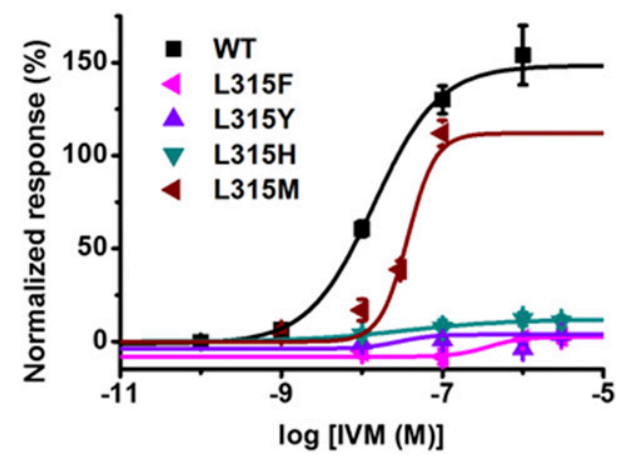

Fig. 9. Responses of Musca L315 mutant GluCls to IVM. (A) Current trace showing the absence of the IVM activation in L315Y GluCls. (B) Current trace when IVM was applied to L315H GluCls. (C) Current trace showing the IVM activation of L315M GluCls. (D) Dose-response curves showing the presence and absence of IVMinduced currents in mutants normalized relative to responses induced by the $\mathrm{EC}_{50}$ values of glutamate. Data points indicate mean \pm S.E.M. values $(n=6)$. acid Thr316 of the Musca GluCl subunit (Fig. 2), adjacent to Leu315, is positionally equivalent to the amino acid that was reported to form a hydrogen bond with IVM in an X-ray crystal study of the C. elegans GluCl- $\alpha$ channel (Hibbs and Gouaux, 2011). The T316V substitution did not change the potency of IVM in the present study (Fig. 3D; Table 1), suggesting that the hydrogen bonding does not contribute substantially to IVM binding in Musca GluCls. Instead, our data suggest that Leu315 is located adjacent to bound IVM. How the substitution of the amino acid Leu315 impairs the IVM-induced activation of GluCls and the IVM-induced potentiation of glutamate responses remains to be examined.

Finally, we generated the Musca RDL subunit with an inverse mutation (the substitution of Phe with Leu at an equivalent position) (Fig. 2A) to evaluate whether this mutation results in low sensitivity of GABACls to fluralaner. However, because this mutation led to a spontaneously open channel, we were unable to determine the potency of fluralaner in this mutant.

In conclusion, we have shown that Leu315 located in the TSI of Musca GluCls and the positionally equivalent amino acid of GABACls play key roles in determining the selectivity of fluralaner and IVM toward these channels. This finding may be applicable to other GluCls and GABACls, given that the TM3 sequence is highly conserved among insect and mite species (Supplemental Fig. 1). As predicted, a very recent publication has indicated that activation by IVM was strongly reduced and that activation by okaramine $\mathrm{B}$, an insecticidal indole alkaloid, was completely abolished in the silkworm (Bombyx mori) GluCl containing an L319F mutation, which is equivalent to the L315F mutation in the Musca GluCl (Furutani et al., 2017). More importantly, we have shown in the present study that the L315F mutation has opposite impacts on the selectivity of fluralaner and IVM for GluCls. This implies that even if IVM-insensitive arthropod pests with an equivalent mutation emerge, these arthropod pests would be sensitive to fluralaner. These findings should prove useful for understanding the modes of action of these parasiticides and further development of pest control agents.

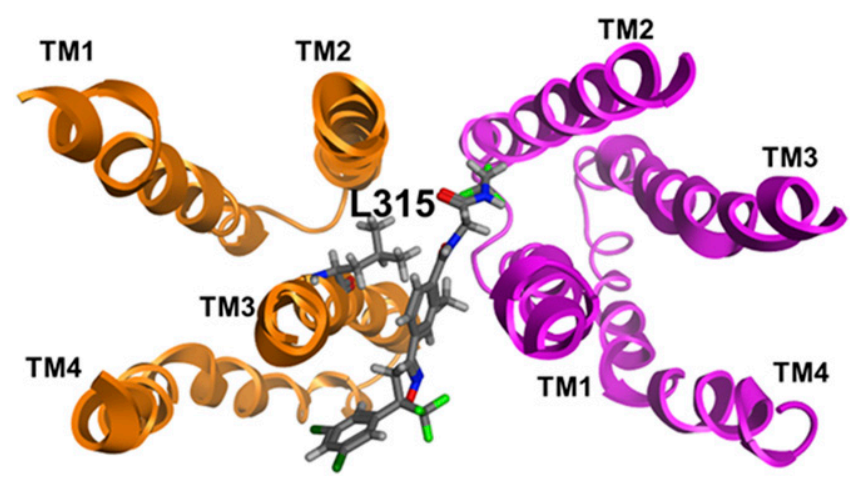

Fig. 10. Docking of the $S$ enantiomer of fluralaner into the TSI of a wild-type Musca GluCl homology model. The $\alpha$-helical transmembrane segments (TM1-TM4) of two adjacent subunits are shown in different colors. The docked fluralaner molecule lies near Leu315, suggesting a $\pi-\pi$ stacking interaction between the phenyl group of fluralaner and the aromatic ring of an amino acid substituted at position 315 of Musca GluCls. The CPK coloring is used for the fluralaner stick model. 


\section{Acknowledgments}

The authors thank T. Kita, K. Nomura, and M. Takashima for technical assistance and advice.

\section{Authorship Contributions}

Participated in research design: Nakata, Asahi, Nakahira, F. Ozoe, and Y. Ozoe.

Conducted experiments: Nakata, Fuse, Yamato, and F. Ozoe.

Performed data analysis: Nakata, Fuse, and Yamato.

Wrote or contributed to the writing of the manuscript: Nakata, Fuse, and Y. Ozoe.

\section{References}

Alexander SPH, Peters JA, Kelly E, Marrion N, Benson HE, Faccenda E, Pawson AJ, Sharman JL, Southan C, and Davies JA; CGTP Collaborators (2015) The concise guide to PHARMACOLOGY 2015/16: ligand-gated ion channels. $\mathrm{Br} J$ Pharmacol 172:5870-5903.

Asahi M, Kobayashi M, Matsui H, and Nakahira K (2015) Differential mechanisms of action of the novel $\gamma$-aminobutyric acid receptor antagonist ectoparasiticides fluralaner (A1443) and fipronil. Pest Manag Sci 71:91-95.

Chen IS, Tateyama M, Fukata Y, Uesugi M, and Kubo Y (2017) Ivermectin activates GIRK channels in a PIP $_{2}$-dependent, $\mathrm{G}_{\beta \gamma}$-independent manner and an amino acid residue at the slide helix governs the activation. J Physiol 595:5895-5912.

Cully DF, Paress PS, Liu KK, Schaeffer JM, and Arena JP (1996) Identification of a Drosophila melanogaster glutamate-gated chloride channel sensitive to the antiparasitic agent avermectin. J Biol Chem 271:20187-20191.

Cully DF, Vassilatis DK, Liu KK, Paress PS, Van der Ploeg LHT, Schaeffer JM, and Arena JP (1994) Cloning of an avermectin-sensitive glutamate-gated chloride channel from Caenorhabditis elegans. Nature 371:707-711.

Dawson GR, Wafford KA, Smith A, Marshall GR, Bayley PJ, Schaeffer JM, Meinke PT, and McKernan RM (2000) Anticonvulsant and adverse effects of avermectin

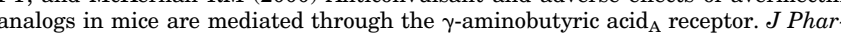
macol Exp Ther 295:1051-1060.

Eguchi Y, Ihara M, Ochi E, Shibata Y, Matsuda K, Fushiki S, Sugama H, Hamasaki Y, Niwa H, Wada M, et al. (2006) Functional characterization of Musca glutamateand GABA-gated chloride channels expressed independently and coexpressed in Xenopus oocytes. Insect Mol Biol 15:773-783.

Estrada-Mondragon A and Lynch JW (2015) Functional characterization of ivermectin binding sites in $\alpha 1 \beta 2 \gamma 2 \mathrm{~L}$ GABA(A) receptors. Front Mol Neurosci 8:55.

Forman SA and Miller KW (2016) Mapping general anesthetic sites in heteromeric $\gamma$-aminobutyric acid type A receptors reveals a potential for targeting receptor subtypes. Anesth Analg 123:1263-1273.

Furutani S, Okuhara D, Hashimoto A, Ihara M, Kai K, Hayashi H, Sattelle DB, and Matsuda K (2017) An L319F mutation in transmembrane region 3 (TM3) selectively reduces sensitivity to okaramine $\mathrm{B}$ of the Bombyx mori L-glutamate-gated chloride channel. Biosci Biotechnol Biochem 21:1-7.

Fuse T, Kita T, Nakata Y, Ozoe F, and Ozoe Y (2016) Electrophysiological characterization of ivermectin triple actions on Musca chloride channels gated by L-glutamic acid and $\gamma$-aminobutyric acid. Insect Biochem Mol Biol 77:78-86.

Gassel M, Wolf C, Noack S, Williams H, and Ilg T (2014) The novel isoxazoline ectoparasiticide fluralaner: selective inhibition of arthropod $\gamma$-aminobutyric acidand L-glutamate-gated chloride channels and insecticidal/acaricidal activity. Insect Biochem Mol Biol 45:111-124.

Hibbs RE and Gouaux E (2011) Principles of activation and permeation in an anionselective Cys-loop receptor. Nature 474:54-60.

Jayakar SS, Zhou X, Savechenkov PY, Chiara DC, Desai R, Bruzik KS, Miller KW, and Cohen JB (2015) Positive and negative allosteric modulation of an $\alpha 1 \beta 3 \gamma 2$ $\gamma$-aminobutyric acid type $\mathrm{A}\left(\mathrm{GABA}_{\mathrm{A}}\right)$ receptor by binding to a site in the transmembrane domain at the $\gamma^{+}-\beta^{-}$interface. J Biol Chem 290:23432-23446.

Krause RM, Buisson B, Bertrand S, Corringer P-J, Galzi J-L, Changeux J-P and Bertrand D (1998) Ivermectin: a positive allosteric effector of the $\alpha 7$ neuronal nicotinic acetylcholine receptor. Mol Pharmacol 53:283-294.

Kwon DH, Yoon KS, Clark JM, and Lee SH (2010) A point mutation in a glutamategated chloride channel confers abamectin resistance in the two-spotted spider mite, Tetranychus urticae Koch. Insect Mol Biol 19:583-591.

Laing R, Gillan V, and Devaney E (2017) Ivermectin—old drug, new tricks? Trends Parasitol 33:463-472.

Lasota JA and Dybas RA (1991) Avermectins, a novel class of compounds: implications for use in arthropod pest control. Annu Rev Entomol 36:91-117.
Lynagh T and Lynch JW (2010) A glycine residue essential for high ivermectin sensitivity in Cys-loop ion channel receptors. Int J Parasitol 40:1477-1481.

McTier TL, Chubb N, Curtis MP, Hedges L, Inskeep GA, Knauer CS, Menon S, Mills B, Pullins A, Zinser E, et al. (2016) Discovery of sarolaner: a novel, orally administered, broad-spectrum, isoxazoline ectoparasiticide for dogs. Vet Parasitol 222:3-11.

Mermans C, Dermauw W, Geibel S, and Van Leeuwen T (2017) A G326E substitution in the glutamate-gated chloride channel $3(\mathrm{GluCl} 3)$ of the two-spotted spider mite Tetranychus urticae abolishes the agonistic activity of macrocyclic lactones. Pest Manag Sci [published ahead of print].

Miller PS and Smart TG (2010) Binding, activation and modulation of Cys-loop receptors. Trends Pharmacol Sci 31:161-174.

Mita T, Kikuchi T, Mizukoshi T, Yaosaka M, and Komoda M (2005) inventors, Nissan Chemical Industries, Ltd., assignee. Isoxazoline-substituted benzamide compound and noxious organism control agent. International patent WO/2005/085216. 2005 Sep 15.

Mita T, Maeda K, Yamada Y, Ikeda E, Toyama K, and Komoda M (2009) inventors, Nissan Chemical Industries, Ltd., assignee. Substituted isoxazoline compound and pest control agent. International patent WO/2009/035004. 2009 Mar 19.

Mita T, Ikeda E, Toyama K, Yamada Y, Iwasa M, and Maeda K (2010) inventors, Nissan Chemical Industries, Ltd., assignee. Substituted acetophenone compound, process for producing same, and use of same. International patent WO/2010/027051. 2010 Mar 11.

Nakao T and Banba S (2016) Broflanilide: a meta-diamide insecticide with a novel mode of action. Bioorg Med Chem 24:372-377.

Nakao T, Banba S, Nomura M, and Hirase K (2013) Meta-diamide insecticides acting on distinct sites of RDL GABA receptor from those for conventional noncompetitive antagonists. Insect Biochem Mol Biol 43:366-375.

Nakatani Y, Furutani S, Ihara M, and Matsuda K (2016) Ivermectin modulation of $\mathrm{pH}$-sensitive chloride channels in the silkworm larvae of Bombyx mori. Pestic Biochem Physiol 126:1-5.

Ömura S and Crump A (2014) Ivermectin: panacea for resource-poor communities? Trends Parasitol 30:445-455.

Ozoe Y (2013) $\gamma$-Aminobutyrate- and glutamate-gated chloride channels as targets of insecticides. Adv Insect Physiol 44:211-286.

Ozoe Y, Asahi M, Ozoe F, Nakahira K, and Mita T (2010) The antiparasitic isoxazoline A1443 is a potent blocker of insect ligand-gated chloride channels. Biochem Biophys Res Commun 391:744-749.

Ozoe Y, Kita T, Ozoe F, Nakao T, Sato K, and Hirase K (2013) Insecticidal 3-benzamido- $N$-phenylbenzamides specifically bind with high affinity to a novel allosteric site in housefly GABA receptors. Pestic Biochem Physiol 107:285-292.

Puinean AM, Lansdell SJ, Collins T, Bielza P, and Millar NS (2013) A nicotinic acetylcholine receptor transmembrane point mutation (G275E) associated with resistance to spinosad in Frankliniella occidentalis. J Neurochem 124:590-601.

Shan Q, Haddrill JL, and Lynch JW (2001) Ivermectin, an unconventional agonist of the glycine receptor chloride channel. J Biol Chem 276:12556-12564.

Shoop WL, Hartline EJ, Gould BR, Waddell ME, McDowell RG, Kinney JB, Lahm GP, Long JK, Xu M, Wagerle T, et al. (2014) Discovery and mode of action of afoxolaner, a new isoxazoline parasiticide for dogs. Vet Parasitol 201:179-189.

Silberberg SD, Li M, and Swartz KJ (2007) Ivermectin interaction with transmembrane helices reveals widespread rearrangements during opening of P2X receptor channels. Neuron 54:263-274.

Silva WM, Berger M, Bass C, Williamson M, Moura DMN, Ribeiro LMS, and Siqueira HAA (2016) Mutation (G275E) of the nicotinic acetylcholine receptor $\alpha 6$ subunit is associated with high levels of resistance to spinosyns in Tuta absoluta (Meyrick) (Lepidoptera: Gelechiidae). Pestic Biochem Physiol 131:1-8.

Smart TG and Paoletti P (2012) Synaptic neurotransmitter-gated receptors. Cold Spring Harb Perspect Biol 4:a009662.

Taenzler J, Wengenmayer C, Williams H, Fourie J, Zschiesche E, Roepke RKA and Heckeroth AR (2014) Onset of activity of fluralaner (BRAVECTO ${ }^{\mathrm{TM}}$ ) against Ctenocephalides felis on dogs. Parasit Vectors 7:567.

Wengenmayer C, Williams H, Zschiesche E, Moritz A, Langenstein J, Roepke RKA and Heckeroth AR (2014) The speed of kill of fluralaner (Bravecto ${ }^{\mathrm{TM}}$ ) against Ixodes ricinus ticks on dogs. Parasit Vectors 7:525.

Wolstenholme AJ, Maclean MJ, Coates R, McCoy CJ, and Reaves BJ (2016) How do the macrocyclic lactones kill filarial nematode larvae? Invert Neurosci 16:7.

Zhao Y, Li J, Gu H, Wei D, Xu YC, Fu W, and Yu Z (2015) Conformational preferences of $\pi-\pi$ stacking between ligand and protein, analysis derived from crystal structure data geometric preference of $\pi-\pi$ interaction. Interdiscip Sci 7:211-220.

Address correspondence to: Dr. Yoshihisa Ozoe, Faculty of Life and Environmental Science, Shimane University, Matsue, Shimane 690-8504, Japan. E-mail: ozoe-y@life.shimane-u.ac.jp 